\title{
ANALYSIS OF IMAGE COMPRESSION ALGORITHMS USING WAVELET TRANSFORM WITH GUI IN MATLAB
}

\author{
Y.Sukanya ${ }^{1}$, J.Preethi ${ }^{2}$ \\ ${ }^{1}$ Associate professor, ${ }^{2} M-T e c h$, ECE, Vignan's Institute Of Information Technology, Andhra Pradesh ,India \\ Sukanya_ece@rediffmail.com,Preetupriti@gmail.com
}

\begin{abstract}
Image compression is nothing but reducing the amount of data required to represent an image. To compress an image efficiently we use various techniques to decrease the space and to increase the efficiency of transfer of the images over network for better access. This paper explains about compression methods such as JPEG 2000, EZW, SPIHT (Set Partition in Hierarchical Trees) and HSSPIHT on the basis of processing time, error comparison, mean square error, peak signal to noise ratio and compression ratio. Due to the large requirement for memory and the high complexity of computation, JPEG2000 cannot be used in many conditions especially in the memory constraint case. SPIHT gives better simplicity and better compression compared to the other techniques. But to scale the image more so as to get better compression we are using the line-based Wavelet transform because it requires lower memory without affecting the result of Wavelet transform. We proposed a highly scalable image compression scheme based on the Set Partitioning in Hierarchical Trees (SPIHT) algorithm. This algorithm is called Highly Scalable SPIHT (HS_SPIHT) it gives good scalability and provides 1 bit stream that can be easily adapted to give bandwidth and resolution requirements.
\end{abstract}

Keywords: - Wavelet transform Scalability, SPIHT, HS-SPIHT, Processing time, Line-based Wavelet transform.

\section{INTRODUCTION}

Compression is the process of reducing large data files into smaller files for efficiency of storage and transmission.

Data compression techniques are:

a. Lossless data compression

b. Lossy data compression

Lossless data compression is nothing but the original data can be reconstructed exactly from compressed data.

Lossy data compression in which data after compression and then decompression retrieves a file that is not exactly as the original data as there will be loss of data.

\subsection{Wavelets Definition}

Wavelets are mathematical functions that cut up data into different frequency components. The fundamental idea behind wavelets is to analyze the signal at different scales or resolutions, which is called multiresolution.

\subsection{Wavelet Transform}

The most important feature of wavelet transform is it allows multiresolution decomposition. An image that is decomposed by wavelet transform can be reconstructed with desired resolution. The procedure for this is a low pass filter and a high pass filter is chosen, such that they exactly halve the frequency range between themselves. This filter pair is called the Analysis Filter pair. First of all, the low pass filter is applied for each row of data, and then we obtain low frequency components of the row. As the LPF is a half band filter, the output data consists of frequencies only in the first half of the original frequency range. By Shannon's Sampling Theorem, they can be sub sampled by two, so that the output data contains only half the original number of samples, similarly the high pass filter is applied for the same row of data, and now the high pass components are separated, and placed by the side of the low pass components. This procedure is done for all rows.

\begin{tabular}{|l|l|}
\hline LL & HL \\
\hline LH & HH \\
\hline
\end{tabular}

Ist level

\begin{tabular}{|l|l|l|}
\hline LL & HL & HL \\
\cline { 1 - 2 } LH & HH & \\
\cline { 1 - 1 } LH & & HH \\
\hline
\end{tabular}

2nd level 


\begin{tabular}{|l|l|l|l|}
\hline LL & HL & HL & \multirow{2}{*}{ HL } \\
\cline { 1 - 2 } LH & HH & & \\
\cline { 1 - 2 } LH & & HH & \\
\cline { 1 - 2 } LH & & HH \\
\hline
\end{tabular}

3rd level

Fig -1: wavelet decomposition.

Next, the filtering is done on each column. as a result we get four bands of data, each labeled as LL (low-low), HL (highlow), LH (low-high) and HH (high-high).The LL band can be decomposed once again in the same manner, thereby producing even more sub bands[4]. This can be done up to any level, thereby resulting in a pyramidal decomposition as shown above the LL band at the highest level can be said as most important, and the other bands are of lesser importance, the degree of importance decreases from the top of the pyramid to bottom.

\section{APPROACH}

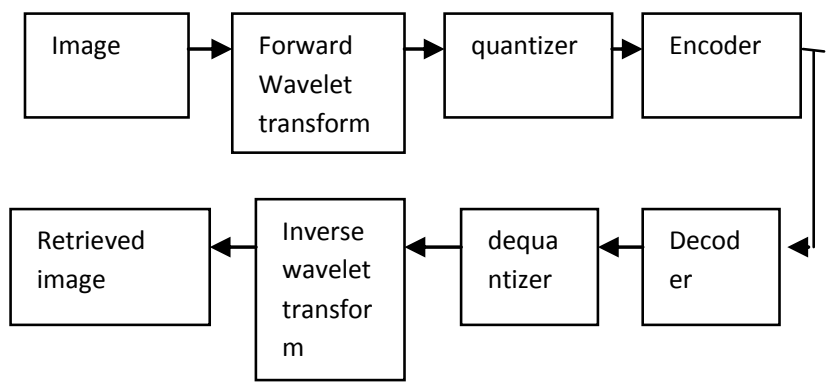

Fig -2: Block diagram for image compression

One of the most important characteristics of DWT is multiresolution decomposition. An image is considered and forward wavelet transforms applied on the image then the image is decomposed the decomposed image is given to the quantizer. The quantizer approximates the continuous set of values in image data with finite set of values. after that data can be encoded using entropy coder to give additional compression. Next is the decoder that carries decoding process i.e. reading the unique code bits sent in place of data bits. the dequantizer dequantizes the decoded data bits then finally the inverse transformation is the process of retrieving back the image data from the obtained image values.

\section{IMAGE COMPRESSION ALGORITHMS}

JPEG 2000

EZW

SPIHT

HS-SPIHT

\section{JPEG-2000 IMAGE CODING}

JPEG 2000 is the international standard for still images. This is the enhancement to the existing JPEG system. The JPEG 2000 implements a new way of compressing images based on the wavelet transform. This supports lossy and lossless compression of grayscale as well as color images. Here we have 2 processes encoding and decoding process.

\subsection{Encoding Process}

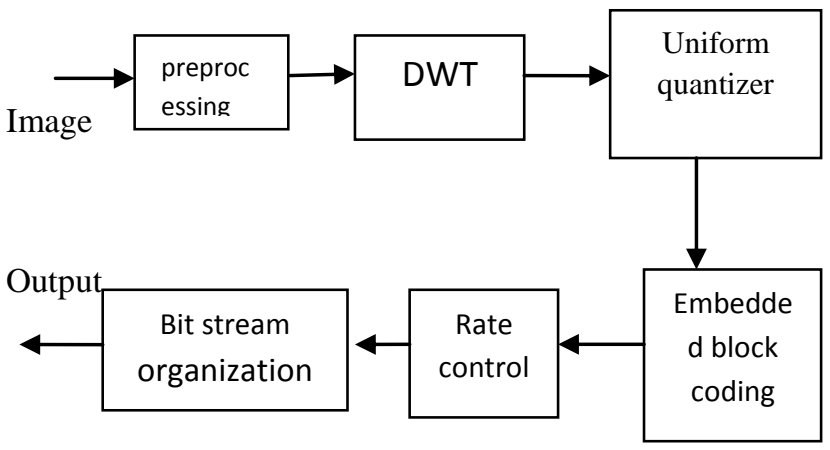

Fig -3: Encoding block diagram of JPEG 2000 algorithm

The input image is given to preprocessor, in this step tiling is done .tiling is nothing but dividing the image into rectangular and non-overlapping tiles of equal size and then each tile is compressed independently using its own set of specified compression parameters. The preprocessed image is given to DWT here wavelet decomposition is done the output of DWT is given to uniform quantizer which reduces precision of sub band coefficients so that fewer bits will be needed to encode transformed coefficients. then output of quantizer is given to embedded block coding which uses wavelet transform to generate the sub band this sub band is partitioned into small blocks of samples called code blocks EBCOT generates a separate highly scalable bit stream for each code block, next is the rate control block which adjusts the quantizer step size or discards some coding pass information

\subsection{Decoding Process}

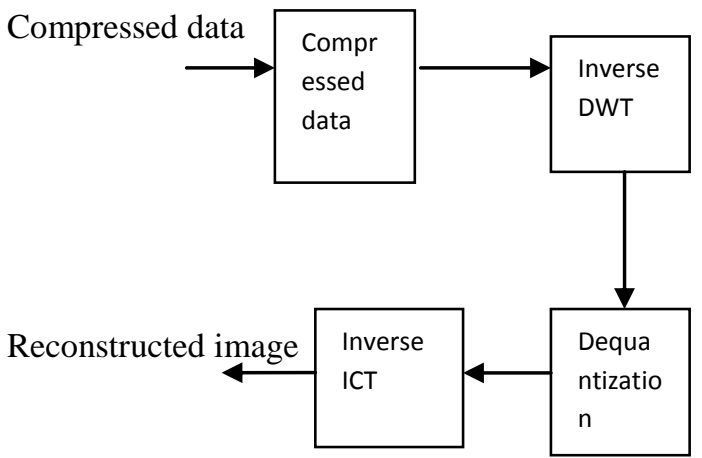

Fig -4: Decoding block diagram of jpeg 2000 
decoder perform opposite to encoder, The output of encoder i.e. the code stream is received by the decoder .the coefficients in packets are decoded, then dequantized and reverse ICT is performed to get reconstructed image.

Important point of jpeg 2000 is here compression can be done in one way but decompression is done in many ways.

\section{EMBEDDED ZERO TREE WAVELET CODING}

EZW stands for embedded zero tree wavelet An EZW encoder is specially designed to use with wavelet transforms. The is based on progressive coding to compress an image into bit stream with increasing accuracy so when more bits are added to a bit stream image will be more detailed.

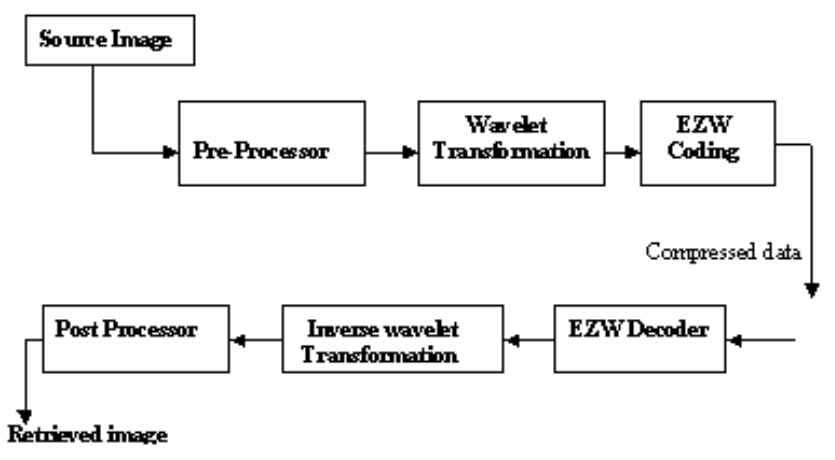

Fig -5: Block diagram of Embedded zero tree wavelet coding

\subsection{Approach}

Before the processing of image data the image is preprocessed .In preprocessing step tiling on the original image is done. All operations, including component mixing, wavelet transform, quantization and entropy coding are performed independently on the image tiles. Tiling reduces memory requirements, and as they are also reconstructed independently, they can be used for decoding specific parts of the image instead of the whole image. All tiles have exactly the same dimensions, except some of those at the boundary of the image. This unit transforms the input image from time domain to frequency domain and decomposes the original image into its fundamental components.

The wavelet transform uses filter banks for the decomposition of preprocessed image. The Embedded ZeroTreeWavelet (EZW) encoder encodes the decomposed image by recognizing the priority of decomposed image pixel. The encoder module calculates a initial threshold for coding given by $\mathrm{T}_{0}=2^{\left(\log _{2} \mathrm{c} \text { max }\right.}$.

The encoding process is performed using 2 passes namely

1. dominant pass and
2. Subordinate pass.

The dominant pass generates any one of four possible combinations like they are significant positive(SP), significant negative (SN), isolated zero(IZ) and zerotree $\operatorname{root}(\mathrm{ZR})$.

Subordinate pass where the coefficients are encoded as 0 or 1 depending on the current threshold.

The decoding unit reconstructs the values by identifying the symbols as positive, negative, zero tree and isolated zero tree. Inverse transformation is the process of retrieving back the image data from the obtained image values. The image data transformed and decomposed under encoding side is rearranged from higher level decomposition to lower level with the highest decomposed level been arranged at the top. Fig 5 shows the reconstruction of the obtained decomposed component.

\subsection{The Algorithm}

1. Set the initial threshold $\mathrm{T}_{0}=2^{\left(\log _{2} \mathrm{c} \text { max }\right.}{ }^{)}$.here $\mathrm{xmax}$ is the maximum coefficient value.

2. set $\mathrm{k}=0$

3. Conduct dominant pass by scanning the data Outputs will be any of 4 conditions below i.e.

a. If value of coefficient is greater than the threshold and the value is positive, it means the output significant positive.

b. If value of coefficient is greater than the threshold and the value is negative, it means the output significant negative.

c. If magnitude of coefficient is less than the threshold and all its descendants have magnitudes less than the threshold then coefficient is labeled as zero tree roots.

d. If magnitude of coefficient is less than the threshold and all its descendants have values greater than the threshold then coefficient is labeled as isolated zero

4. Conduct a subordinate pass or refinement pass by scanning through the data to refine the pixels already known to be significant in current bit plane.

5. Set $\mathrm{k}=\mathrm{k}+1$ and the threshold $\mathrm{T}_{\mathrm{k}}=\mathrm{T}_{\mathrm{k}^{-1}} / 2$.

6. Stop if the stopping criterion is met or go to step 3.

\section{SPIHT ALGORITHM}

Set Partioning in Hierarchical Trees (SPIHT) is a wavelet based image compression method.

SPIHT introduces three lists:

a. List of Significant Pixels (LSP),

b. List of Insignificant Pixels (LIP) and

c. List of Insignificant Sets (LIS).

First initialization is done, and then algorithm takes two stages for each level of threshold 
1. The sorting pass (in which lists are organized) and

2. The refinement pass.

LIS is further divided into two types of sets of insignificant pixels .They are

Type A (all descendant are zero)

Type B (all grandchildren and further descendants are zero).

SPIHT algorithm defines four types of sets, which are sets of coordinates of coefficients:

$\mathrm{O}(\mathrm{i}, \mathrm{j})$ : set of coordinates of all offspring of node $(\mathrm{i}, \mathrm{j})$; children only

D $(i, j)$ : set of coordinates of all descendants of node $(i, j)$; children, grandchildren, great-grand, etc.

$H(i, j)$ : set of all tree roots (nodes in the highest pyramid level); parents

$\mathrm{L}(\mathrm{i}, \mathrm{j}): \mathrm{D}(\mathrm{i}, \mathrm{j})$ - O(i,j) (all descendants except the offspring); grandchildren, great-grand, etc.

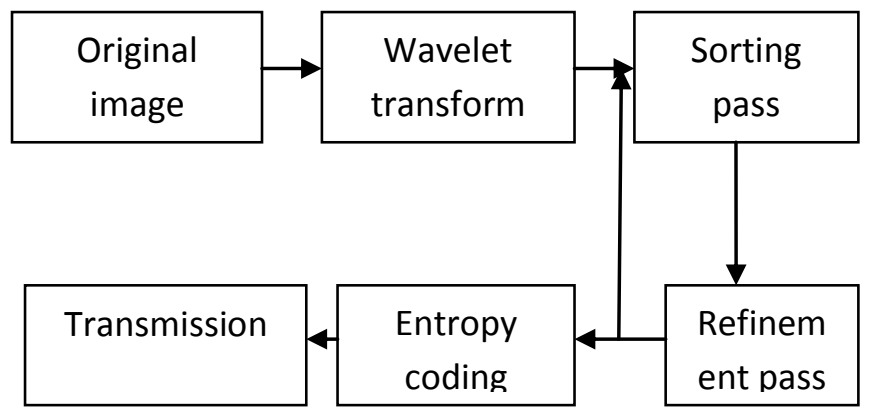

Fig -6: Block diagram of SPIHT

To find the number of passes we use the formula

$$
\mathrm{n}=\left[\begin{array}{l}
\log \mathrm{c} \\
2 \max
\end{array}\right] .
$$

We find initial threshold as $\mathrm{T}_{0}=2^{\mathrm{n}}$.

The SPIHT algorithm forms a hierarchical quad tree data structure for the wavelet transformed coefficients. The set of root node and corresponding descendants are together called as spatial orientation tree (SOT).

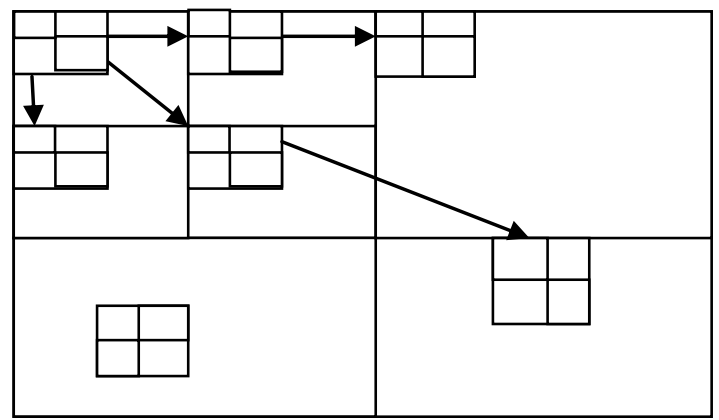

\section{Fig -7: Quad tree structure \\ 7. LINE- BASED WAVELET TRANSFORM:}

In general in wavelet transform all the lines are horizontally filtered before vertical filtering and thus the total image data should be stored. But the line-based wavelet transform starts vertical filtering as soon as a sufficient number of lines, determined by the filter length have been horizontally filtered. This algorithm requires storing a minimum number of lines. The image data are stored in memory only while they are used to generate output coefficients, and removed from memory immediately once no longer being needed, therefore the memory requirements are greatly reduced.

\section{HS_SPIHT ALGORITHM}

The HS_SPIHT algorithm proposed here solves the spatial scalability problem by introducing of resolution-dependent sorting pass that uses one additional list, called the list of delayed insignificant sets (LDIS). The HS_SPIHT coder first encodes all bit planes for a given (low) resolution level and then moves to the next higher resolution level

Sets encountered during the sorting pass that lie outside the actually considered spatial resolution are temporarily stored in the LDIS. They are moved back from the LDIS into the LIS when they are required for encoding the next higher resolution. According to the magnitude of the coefficients in the wavelet pyramid, coding of higher resolution bands usually starts from lower bit planes. Therefore, during the encoding process of resolution level $\mathrm{k}$, the encoder keeps the number of coefficients that went to the LDIS for each quantization level.

After finishing the encoding process for all bit places of resolution level $\mathrm{k}$, the encoder knows which entries in the LDIS that belong to which bit plane. To encode the additional three sub bands for resolution level $\mathrm{k}-1$, it moves the related entries of the LDIS that belong to the actual bit plane to the LIS and carries out the sorting of LIS with the same procedure as before.

Altogether, the total number of bits belonging to a particular bit plane is the same for SPIHT and HS_SPIHT, but HS_SPIHT distributes them differently among the different spatial resolution levels.

\subsection{The Algorithm}

The HS-SPIHT algorithm also uses three lists they are 1. LIP

2. LSP

3. LIS

Algorithm consists of both encoding and decoding process. Encoding:

Step 1: initialization. 
Choose threshold $\mathrm{T}_{0}=2\left[{ }^{\log \mathrm{c}} \max \right]$

Step 2:load the LIP with $\{(0,0),(0,1),(1,0),(1,1)\}$

Step 3:load LIS with descendants i.e. $\{\mathrm{D}(0,1), \mathrm{D}(1,0), \mathrm{D}(1,1)\}$.

Step 4: LSP=Empty.

Step 5: Process LIP

If $(0,0)>\mathrm{T} 0$,we transmit 1 and load LSP with(0,0); if

$(0,0)>0$ we transmit 0 .

Step 6: Process LIS if

$\mathrm{D}(0,1), \mathrm{D}(1,0), \mathrm{D}(1,1)<\mathrm{T} 0$ transmit 0 .

We get a bit stream

Decoding:

Step 1: Initialization

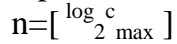

Step 2: Find threshold $\mathrm{T} 0=2^{\mathrm{n}}$.

Step 3: consider LIP, LSP, LIS

Step 4: consider the encoded bit steam

Step 5: Process LIP

Receive the bit stream, if 1 st bit is 1 combine next bit to 1

i.e.10 this indicates ist element of LIP is significant positive.
If next bit is 0 we it as insignificant

Step 6: reconstruct the 1 st element of LIP value by( $3 / 2) * 2^{n}$.

Step 6: if $\mathrm{D}(0,1), \mathrm{D}(1,0), \mathrm{D}(1,1)<\mathrm{T} 0$ we get 0

Step 7: Finally again load LIP, LSP, LIS.

\section{RESULTS}

\section{For Camera Man Image}

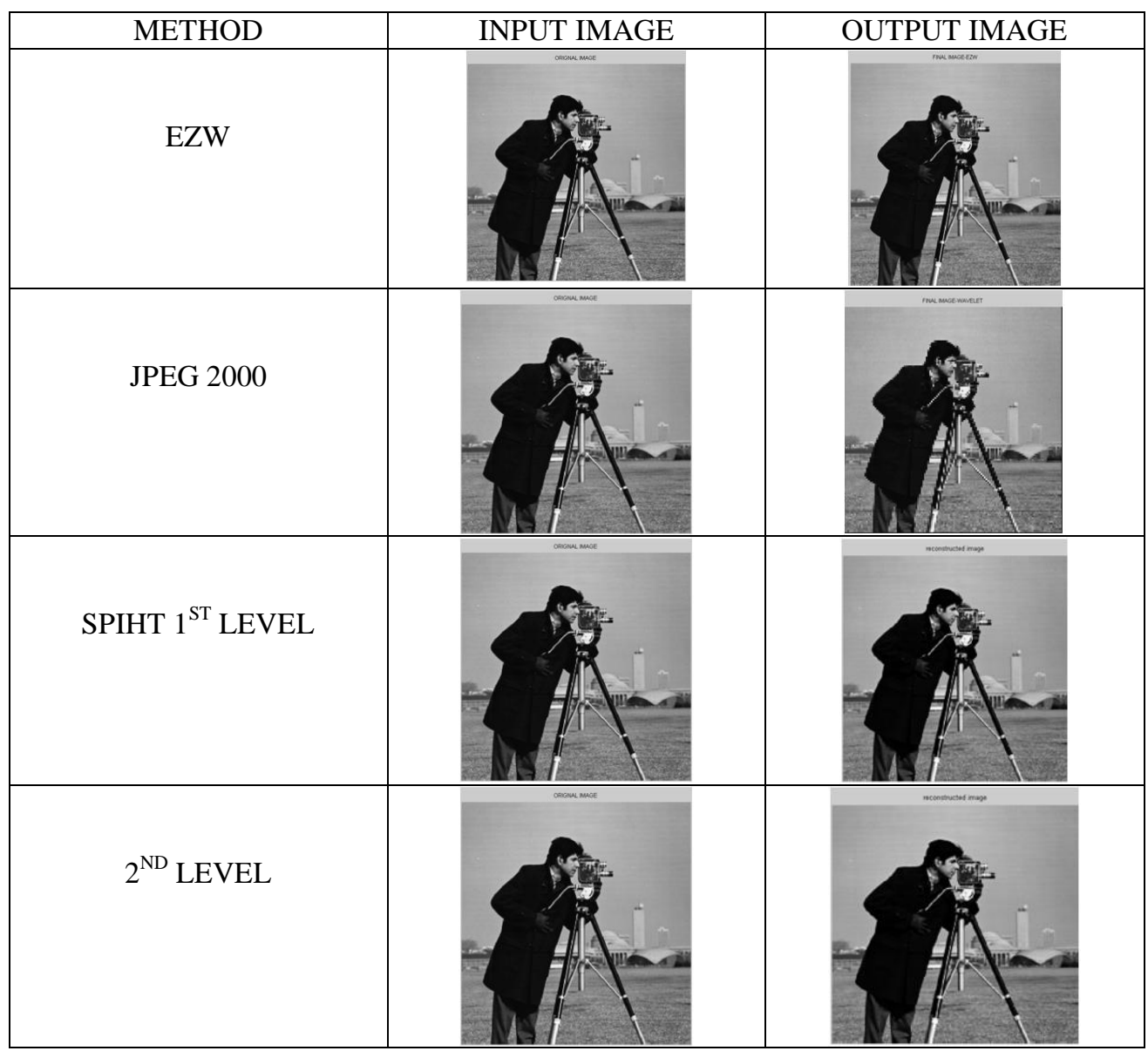




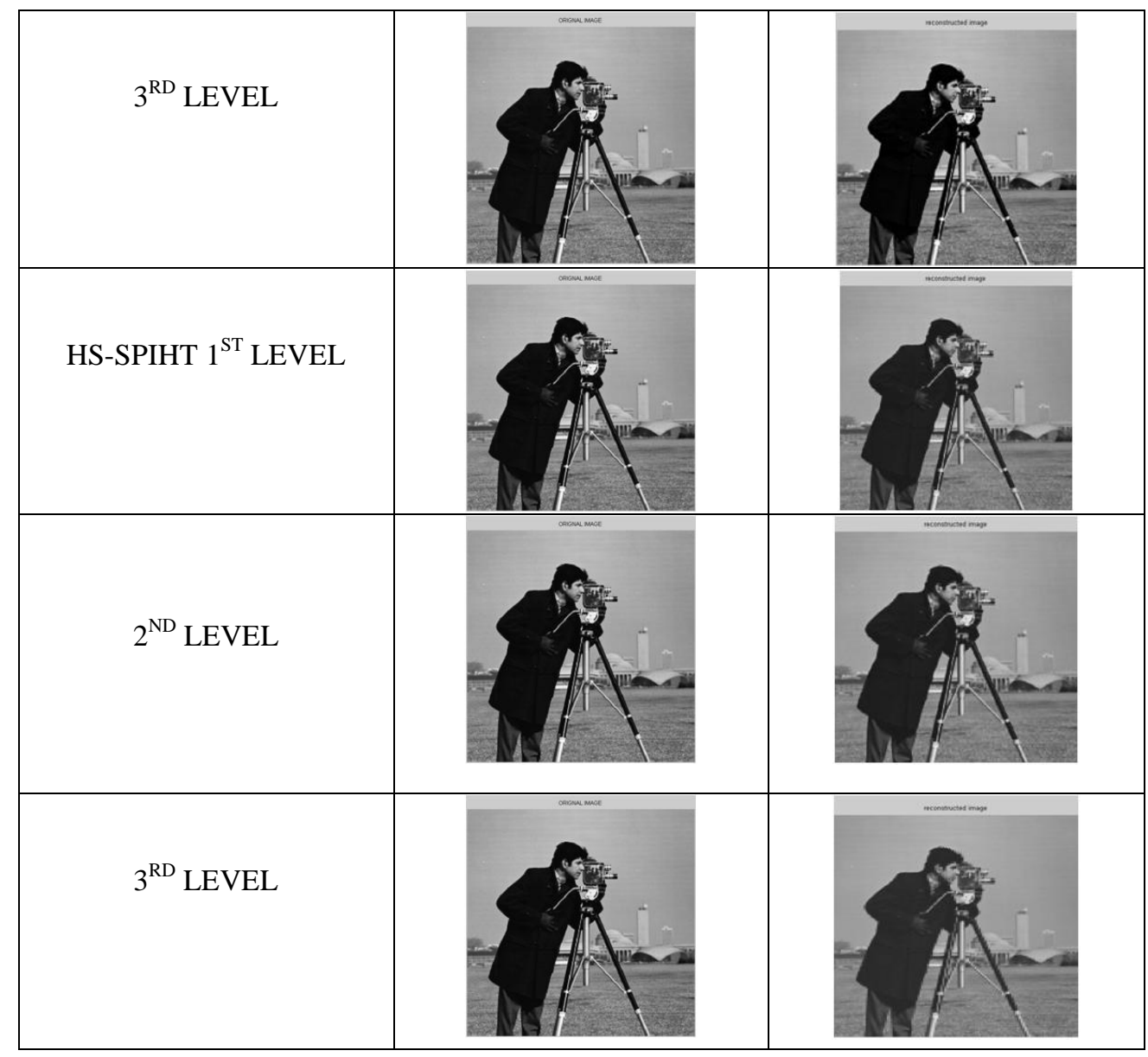

Table:

\begin{tabular}{|c|c|c|c|c|c|c|c|}
\hline METHOD & & $\begin{array}{l}\text { MEMORY } \\
\text { IN MB }\end{array}$ & SIZE & MSE & PSNR & $\begin{array}{c}\text { PROCESSING } \\
\text { TIME }\end{array}$ & $\begin{array}{c}\text { COMPRESION } \\
\text { RATIO }\end{array}$ \\
\hline EZW & & 3.064 & $1 * 290493$ & 0.23 & 30.4481 & 1.9 & 4.44 \\
\hline $\begin{array}{l}\text { JPEG } \\
2000\end{array}$ & & 1.859 & $1 * 204930$ & 0.26 & 29.9157 & 1.12 & 4.46 \\
\hline \multirow{3}{*}{ SPIHT } & $\begin{array}{c}1^{\text {st }} \\
\text { level }\end{array}$ & 3.4 & $1 * 425988$ & \multirow{3}{*}{0.23} & \multirow{3}{*}{30.4481} & \multirow{3}{*}{40.6} & 12.595 \\
\hline & $\begin{array}{l}2^{\text {nd }} \\
\text { level }\end{array}$ & 3.61 & $1 * 451808$ & & & & 13.2009 \\
\hline & $\begin{array}{c}3^{\text {rd }} \\
\text { level }\end{array}$ & 3.84 & $1 * 480133$ & & & & 13.4692 \\
\hline \multirow{3}{*}{$\begin{array}{l}\text { HS- } \\
\text { SPIHT }\end{array}$} & 1stlevel & 3.818 & $1 * 439871$ & \multirow{3}{*}{0.68} & \multirow{3}{*}{29.76} & \multirow{3}{*}{39.45} & 13.9535 \\
\hline & $\begin{array}{l}2^{\text {nd }} \\
\text { level }\end{array}$ & 3.648 & $1 * 456117$ & & & & 16.9535 \\
\hline & $\begin{array}{l}3^{\text {rd }} \\
\text { level }\end{array}$ & 3.875 & $1 * 484442$ & & & & 16.2625 \\
\hline
\end{tabular}




\section{For Medical Image:}

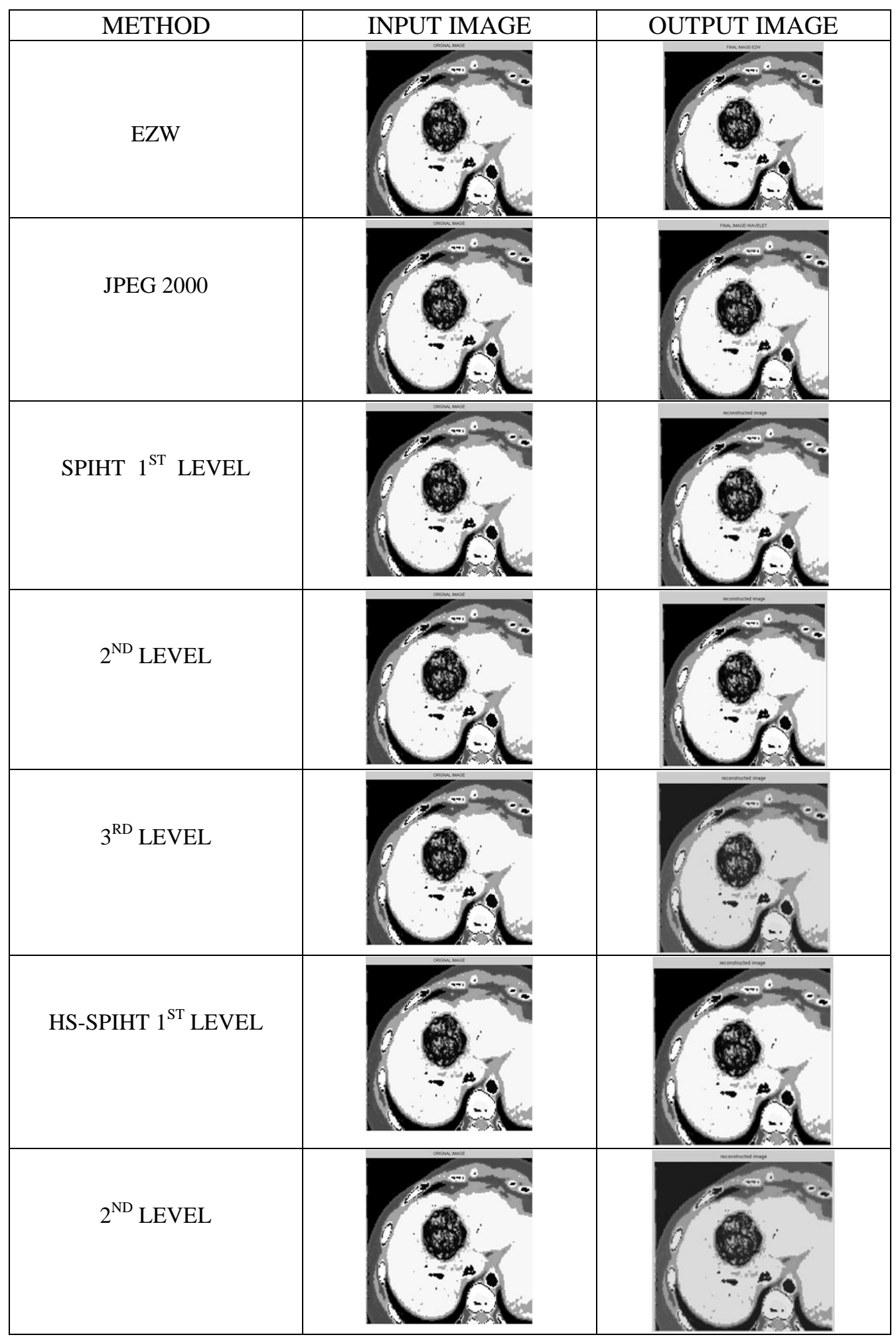




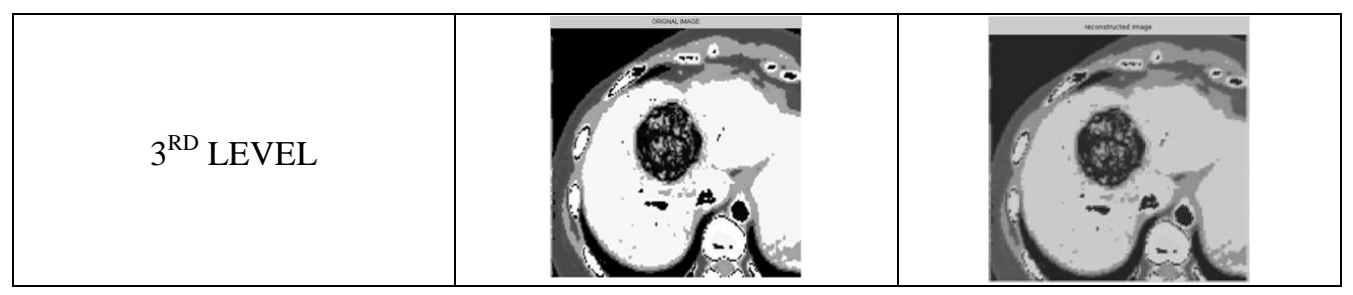

Table

\begin{tabular}{|c|c|c|c|c|c|c|c|}
\hline METHOD & & SIZE & $\begin{array}{l}\text { MEMORY } \\
\text { IN MB }\end{array}$ & MSE & PSNR & $\begin{array}{l}\text { PROCESSING } \\
\text { TIME }\end{array}$ & $\begin{array}{l}\text { COMPRESSION } \\
\text { RATIO }\end{array}$ \\
\hline EZW & & $1 * 198828$ & 1.5 & 0.23 & 30.448 & 3 & 4.43 \\
\hline $\begin{array}{l}\text { JPEG } \\
2000\end{array}$ & & $I * 202692$ & 1.62 & 0.54 & 26.74 & 138.67 & 8.66 \\
\hline \multirow[t]{3}{*}{ SPIHT } & $\begin{array}{l}1^{\text {st }} \\
\text { level }\end{array}$ & $1 * 384046$ & 0.27 & \multirow[t]{3}{*}{0.13} & \multirow[t]{3}{*}{56.958} & \multirow[t]{3}{*}{38.796} & 15.3782 \\
\hline & $\begin{array}{l}2^{\text {nd }} \\
\text { level }\end{array}$ & $1 * 408208$ & 0.261 & & & & 16.0181 \\
\hline & $\begin{array}{l}3^{\text {rd }} \\
\text { level }\end{array}$ & $1 * 436167$ & 0.257 & & & & 16.2924 \\
\hline \multirow[t]{3}{*}{$\begin{array}{c}\text { HS- } \\
\text {-SPIHT }\end{array}$} & $\begin{array}{l}1^{\text {st }} \\
\text { level }\end{array}$ & $1 * 428931$ & 0.40 & \multirow[t]{3}{*}{1.15} & \multirow[t]{3}{*}{23.4547} & \multirow[t]{3}{*}{50.391} & 10.3561 \\
\hline & $\begin{array}{l}2^{\text {nd }} \\
\text { level }\end{array}$ & $1 * 404991$ & 0.24 & & & & 10.3540 \\
\hline & $\begin{array}{l}3^{\text {rd }} \\
\text { level }\end{array}$ & $1 * 482417$ & 0.227 & & & & 18.4595 \\
\hline
\end{tabular}

\section{CONCLUSIONS}

- In this paper Image is considered and wavelet transform is applied on the image and wavelet decomposition is done.

- Four algorithms in terms memory spacing, size, compression ratio, mean square error, peak signal to noise ratio are analyzed.

- We proposed HS-SPIHT algorithm which gives us better scalability and reduced bit stream i.e. size of image is reduced well and it also gives better compression ratio .

- So this paper presents comparative analysis between compression algorithms. In this work we have provided the basics of wavelet transform and comparisons of different algorithms used for an image.

- Finally we get reduced bit stream and better scalability.

\section{REFRENCES}

[1]. Puja D Saraf, Deepti Sisodia, Amit Sinhal and Neetesh Gupta, "Comparisons of wavelets based image compression methods",World Journal of Science and Technology 2012, 2(3):10-13 ISSN: 2231 - 2587.
[2]. D.Vijendra babu , Dr.N.R.Alamelu "Wavelet Based Medical Image Compression Using ROI EZW", International Journal of Recent Trends in Engineering, Vol 1, No. 3, May 2009.

[3].K.Sivanagireddy,M.Saipravallika,P.K.Chandrika

Tejaswini, "Low memory low complexity image compression using DWT and HS-SPIHT encoder ",International Journal of Scientific \& Engineering Research Volume 3, Issue 8, August2012.

[4]. Ruchika, Mooninder Singh, Anant Raj Singh "Compression of Medical Images Using Wavelet Transforms"

, International Journal of Soft Computing and Engineering (IJSCE) ISSN: 2231-2307, Volume-2, Issue-2, May 2012.

[5] .Peng Jiang, Qingbo Huang, Yaguang Kang and Li Chai, "Research on Compression Method based on IWT and SPIHT for Historical Data in Process Industry", Computer and Computational Sciences 2006, Proceedings (IMSCCS'06).

[6] . J.M. Shapiro, "Embedded image coding using the zero trees of wavelet coefficients", IEEE Transactions on ImageProcessing, Vol. 41, No. 12, pp. 3445-3462, December 1993.

[7]. Yen-Yu Chen*, “ Medical image compression using DCT-based subband decomposition and modified SPIHT data organization", international journal of medical informatics 76

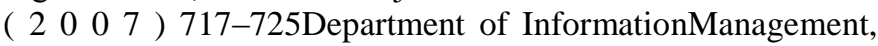


ChengChou Institute of Technology, 6, Line 2, Sec 3, ShanChiao Rd., Yuanlin, Changhwa, Taiwan.

[8]. W. Pennebaker and J. Mitchell, JPEG Still Image Data Compression Standard. Van Nostrand Reinhold, 1994.

[9]. Subramanya A, "Image Compression Technique,’Potentials IEEE, Vol. 20, Issue 1, pp 19-23, FebMarch2001,

[10]. David Jeff Jackson \& Sidney Joel Hannah, "Comparative Analysis of image Compression Techniques," System Theory 1993, Proceedings SSST'93, 25th Southeastern Symposium,pp 513-517, 7 -9 March 1993.

\section{BIOGRAPHIES}

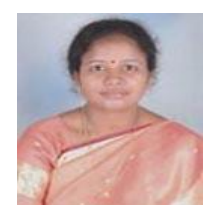

Mrs. Y. Sukanya received her B. Tech Degree in ECE Department from S.R.K.R Engineering College, Bheemavaram and obtained her M.Tech degree in Digital Electronics and Communication Systems from Gudlavalleru Engineering College. She has eight years of teaching experience, currently working at Vignan's Institute of Information Technology, Visakhapatnam as Associate Professor in Department of ECE. Her Area of interests are Communication systems and signal processing.

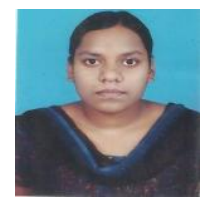

Ms. J.Preethi has obtained B.Tech degree from Vignan's institute of information technology affiliated to JNTUK in the year 2011. Now she is pursuing M.Tech Degree in Department of Electronics \& Communications, Vignan's institute of Information and Technology, Visakhapatnam. She is interested in the fields of image processing. 